INPLASY

PROTOCOL

To cite: Zhang et al.

Psychotherapy for depression in college students: a protocol for systematic review and network meta-analysis. Inplasy protocol 202070134. doi: 10.37766/inplasy2020.7.0134

Received: 31 July 2020

Published: 31 July 2020

Corresponding author: Lin Wan

390052783@qq.com

Author Affiliation:

Third Ward of Orthopedics Department, The Second Hospital of Lanzhou University

Support: GSWSKY-2019-102; CY2018-HL18

Review Stage at time of this submission: Preliminary searches.

Conflicts of interest: There are no potential conflicts of interest to disclose.

\section{Psychotherapy for depression in college students: a protocol for systematic review and network meta-analysis}

Zhang, X1; Niu, MM²; Ma, PF3; Du, L4; Wan, L55.

Review question / Objective: To evaluate depressive symptoms, life satisfaction, self-confidence, substance use, social adjustment, and dropout rates of the use of psychological intervention for college students.

Condition being studied: According to the World Health Organization, more than $\mathbf{3 5 0}$ million people worldwide suffer from depression. The current incidence of depression in China is $6.1 \%$. Because college students are faced with complex self and social problems, coupled with heavy learning tasks, they are prone to strong psychological conflicts, which can lead to depression. So compared with their peers, college students have a higher risk of depression.

INPLASY registration number: This protocol was registered with the International Platform of Registered Systematic Review and Meta-Analysis Protocols (INPLASY) on 31 July 2020 and was last updated on 31 July 2020 (registration number INPLASY202070134).

\section{INTRODUCTION}

Review question / Objective: To evaluate depressive symptoms, life satisfaction, self-confidence, substance use, social adjustment, and dropout rates of the use of psychological intervention for college students. 
Condition being studied: According to the World Health Organization, more than 350 million people worldwide suffer from depression. The current incidence of depression in China is $6.1 \%$. Because college students are faced with complex self and social problems, coupled with heavy learning tasks, they are prone to strong psychological conflicts, which can lead to depression. So compared with their peers, college students have a higher risk of depression.

\section{METHODS}

Search strategy: The search terms will include "depression", "depressive disorder", "students", "universi*, "college". The following sources will be searched: PubMed, Embase, Web of Science, The Cochrane Library. We will also search Clinical Trials. gov, the WHO International Clinical Trials Registry Platform for unpublished data.

Participant or population: The patients we will include are college students diagnosed with depression according to any diagnostic criteria, such as Diagnostic and Statistical Manual of Mental Disorders (DSM)-III, DSM -IV, and International Classification of Diseases, 10th Revision (ICD-10).

Intervention: For psychotherapy, cognitivebehavioral therapy (CBT), mindfulness therapy, mindfulness meditation therapy, comprehensive self-control training (CSCT), interpersonal psychotherapy (IPT), acceptance and commitment therapy (ACT) will be included.

Comparator: In terms of control conditions, waiting-list control (WLC), non-treatment control, physical exercise, bibliotherapy, treatment as usual (TAU) will be included.

Study designs to be included: Any relevant randomized controlled trials (RCTs) will be included.

Eligibility criteria: The eligibility criteria will be the following:1. Types of study: Randomized controlled trials (RCTs). 2.
Participants: College students diagnosed with depression. 3.Interventions: Treatment strategies include: cognitive-behavioral therapy (CBT), mindfulness therapy, mindfulness meditation therapy, comprehensive self-control training (CSCT), interpersonal psychotherapy (IPT), acceptance and commitment therapy (ACT). Controlled interventions will include: waiting-list control (WLC), non-treatment control, physical exercise, bibliotherapy, treatment as usual (TAU).

Information sources: We will identify relevant trials from systematic searches in the following electronic databases: PubMed, Embase, Web of Science, The Cochrane Library. We will also search Clinical Trials. gov, the WHO International Clinical Trials Registry Platform for unpublished data.

Main outcome(s): The primary outcome is depressive symptoms that means the change in severity of depression from baseline to end point which is measured by the depression scale, such as Beck Depression Inventory (BDI), The Center for Epidemiologic Studies Depression Scale (CESD-R), Hamilton Rating Scale for Depression (HRSD). The second outcomes include self-confidence, life satisfaction, substance use, social adjustment and dropout rates.

Quality assessment / Risk of bias analysis: According to Cochrane Collaboration's Risk of bias tool, We will assess risk of bias as 'low risk', 'unclear risk' or 'high risk'. The following items will be evaluated: sequence generation, allocation concealment, blinding of participants and personnel, blinding of outcome assessors, incomplete outcome data, selective outcome reporting and other sources of bias. The evaluation will be conducted by two independent raters. Any disagreements will be resolved by a third review author.

Strategy of data synthesis: We will use Stata V.15.0. software to map network plot to compare the effects of interventions. We will use relative risk (RR) for the dichotomous data, use standard mean 
differences (SMD) for the continuous outcome and calculate the $95 \% \mathrm{Cl}$.

Subgroup analysis: If statistical heterogeneity is evident, we will analyze the causes of heterogeneity, if there is enough data.

Sensibility analysis: We will use the exclusion method to conduct sensitivity analysis: (1) exclude low-quality studies; (2) exclude studies with comorbid physical or mental illnesses; (3) exclude trials with missing data.

Language: English.

Country(ies) involved: China.

Keywords: Depression; College students; Psychotherapy; Network meta-analysis.

Contributions of each author:

Author 1 - Xiu Zhang - The author designed this protocol and developed the search strategies.

Author 2 - Mingming Niu - The author drafted the protocol and contributed to the extraction of research data.

Author 3 - Peifen Ma - The author contributed to the extraction of research data and evaluation of bias.

Author 4 - Li Du - The author contributed to evaluation of bias and analysis of results.

Author 5 - Lin Wan - The author read, provided feedback and approved the final manuscript. 